\title{
RELATIONS HOTES-PARASITE DU TRÉMATODE MICROPHALLUS PAPILLOROBUSTUS (RANKIN, 1940)
}

\author{
I. Pénétration des cercaires et rapports des métacercaires \\ avec le tissu nerveux des Gammarus, hôtes intermédiaires
}

\author{
S. HELLUY*(1)
}

RÉSUMÉ. Les cercaires de Microphallus papillorobustus infestent les Gammares (Gammarus insensibilis et G. aequicauda) en traversant la cuticule des lamelles branchiales; elles forment à la surface de ces dernières des kystes de pénétration.

Les métacercaires s'enkystent dans les ganglions cérébroïdes ou dans la chaîne nerveuse ventrale et les nerfs métamériques. Dans le microhabitat cérébral les larves, quand elles sont peu nombreuses, sont situées principalement dans le protocérébron.

\section{Host-parasite interfaces of the Trematode Microphallus papillorobustus} (Rankin, 1940). I. Penetration of cercariae and relationship of metacercariae with the nervous system in Gammarus, intermediate hosts.

SUMMARY. Microphallus papillorobustus cercariae enter the gills of Gammarids (Gammarus insensibilis and G. aequicauda) through the branchial cuticle; they form penetration cysts on their surface.

Metacercariae encyst in cerebroid ganglions or ventral nervous chain and metameric nerves. In the cerebral microhabitat, larvae are mainly located in the protocerebrum unless present in large numbers.

\section{Introduction}

Dans les eaux saumâtres du littoral languedocien, Microphallus papillorobustus (Rankin, 1940) infeste au stade métacercaire deux espèces-sœurs sympatriques de Gammarus (Crustacea : Amphipoda) appartenant au groupe Locusta : G. insensibilis Stock, 1966 et G. aequicauda (Martynov, 1931).

* Laboratoire de Parasitologie comparée. Université des Sciences et Techniques du Languedoc, Place Eugène Bataillon, F 34060 Montpellier Cedex.

Ce travail a été effectué dans le cadre de l'Équipe de Recherche * Écologie des Plathelminthes parasites " de 1'Université de Montpellier II et de 1'Université de Perpignan, associée au C.N.R.S. (E.R.A. no $9 \mathrm{I} 5$ ).

(I) Avec la coopération technique de Madame Euzet, S., technicienne C.N.R.S. au laboratoire de Parasitologie comparée.

Accepté le I7 décembre I98I. 
Il s'est avéré que les larves de $M$. papillorobustus s'enkystent dans des microhabitats différents (ganglions cérébroïdes ou chaîne nerveuse) en fonction de l'espèce et de l'âge du Gammarus-hôte. De plus, les métacercaires matures localisées dans le cerveau, induisent chez ces Amphipodes de profondes modifications de comportement qui les rendent plus accessibles aux Oiseaux, hôtes définitifs de $M$. papillorobustus. La transmission du Ver est ainsi favorisée. Ces aspects des relations hôtesparasite de $M$. papillorobustus seront traités dans un article ultérieur*. Le présent travail se propose d'étudier le processus de pénétration des cercaires et les rapports des métacercaires avec le tissu nerveux des Gammares.

\section{Matériel et techniques}

Le cycle biologique de $M$. papillorobustus a été élucidé par Rebecq (1964) en Camargue. D'après cet auteur les Gastéropodes Hydrobia acuta (Draparnaud, 1805) et $H$. ventrosa (Montagu, 1803) (Prosobranches, Hydrobiidae) hébergent les premiers stades larvaires du parasite ; Gammarus locusta s.l. abrite les métacercaires. Vingtneuf espèces d'Oiseaux, principalement des Charadriidae mais aussi des Laridae et des Anatidae, sont connues comme hôtes définitifs de $M$. papillorobustus.

Depuis le taxon Gammarus locusta a été scindé en plusieurs espèces (Stock, 1967). Celles présentes dans les eaux saumâtres méditerranéennes sont Gammarus insensibilis et Gammarus aequicauda (Brun, 1971).

Hydrobia et Gammarus sont récoltés à l'épuisette au bord des étangs. La pénétration des cercaires est suivie chez de jeunes Gammares au microscope optique.

Des coupes sériées ( $7 \mu \mathrm{m}$ d'épaisseur) de têtes d'Amphipodes ont été effectuées après fixation dans le Bouin-Hollande pendant 5 jours. La technique de coloration utilisée est celle de Cleveland-Wolf (Glychemalum, éosine $1 \%$, orange G, bleu d'aniline).

Les têtes de Gammares coupées à l'ultra-microtome ont été traitées suivant les techniques classiques utilisées en microscopie électronique à transmission (double fixation au glutaraldéhyde et à l'acide osmique et rinçage dans un tampon de cacodylate de sodium). Les coupes semi-fines (1 $\mu \mathrm{m}$ d'épaisseur) déposées sur lame sont colorées au bleu de méthylène.

\section{Pénétration des cercaires.}

\section{Résultats}

Les xiphidiocercaires (décrites par Rebecq, 1964) nagent sans orientation précise et restent indifférentes au Gammare même quand elles le touchent accidentellement en un point quelconque du corps. Par contre, si elles sont prises par le courant d'eau circulaire engendré par le battement des pléopodes ( $f i g .1$ ), elles sont projetées sur

* Helluy S. - Relations hôtes-parasite du Trématode Microphallus papillorobustus (Rankin, I940). II. Modifications du comportement des Gammarus, hôtes intermédiaires et localisation des métacercaires. (à paraître). 


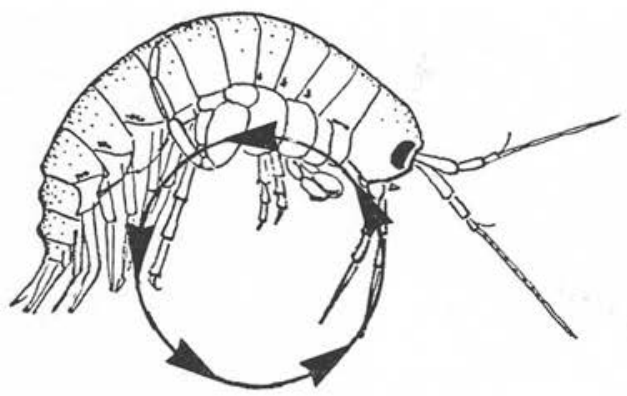

Fig. I. - Courant d'eau engendré par le battement des pléopodes du Gammare.

les lamelles branchiales* et tentent de s'y accrocher (elles ne manifestent apparemment pas de rhéotactisme positif). Certaines y parviennent mais d'autres sont reprises par le courant respiratoire qui les ramène entre les péréiopodes. Le Gammare réagit à la présence des cercaires sur ses lobes branchiaux en " ruant », ce qui contribue à décrocher quelques larves. Toutefois, j'ai pu assister à une douzaine de pénétrations qui toutes ont eu lieu dans les lamelles branchiales. Des cercaires mises en présence de lamelles isolées ne tentent ni de s'en approcher ni de s'y engager ; elles ne semblent pas obéir à un chimiotactisme positif. Le choc serait donc le stimulus nécessaire au déclenchement du processus de pénétration. Lorsque la cercaire adhère par la ventouse orale à la cuticule branchiale, elle perd sa queue. Elle forme alors un pseudokyste (Prevot, 1974) soudé à la cuticule au niveau de la ventouse orale et présentant la forme du corps cercarien (fig. 2). Le pseudo-kyste mesure approximativement $90 \times 55 \mu \mathrm{m}$ et sa paroi atteint $7 \mu \mathrm{m}$ d'épaisseur. Lors de la formation du kyste de pénétration, pour l'observateur tout se passe comme si la cercaire se débarrassait d'abord d'une mince "cuticule externe " qui s'épaissit ensuite de manière centripète par adjonction de substances émises par les glandes cutanées couvrant le corps.

\section{Discussion}

Nos observations diffèrent donc de celles de Rebecq (1964) : " C'est autour de la tête que le " désir " des cercaires est le plus manifeste... nous avons pu voir une cercaire s'engager à la base de l'antenne gauche ".

Par contre, les processus d'infestation observés sont analogues à ceux étudiés par Prevot (1974). Microphallus bittii, Megalophallus carcini et Gynaecotyla longiintestinata pénètrent chez Carcinus mediterraneus au niveau des branchies. Les cercaires de ces Microphallides sont amenées dans la chambre branchiale par le courant respiratoire du Crabe; il en est de même pour Microphallus pachygrapsi chez Pachygrapsus marmoratus. Les cercaires de $M$. carcini et de $G$. longiintestinata sécrètent une capsule ou pseudo-kyste coiffant toute la face dorsale de la cercaire, la face ventrale étant en contact avec la cuticule de la branchie. Dans le cas de $M$. papillorobustus, l'ancrage n'est réalisé qu'autour de la ventouse orale.

* Gammarus aequicauda et $G$. insensibilis possèdent 6 paires de lamelles branchiales prenant naissance sur les coxopodites des péréiopodes 2 à 7 . 

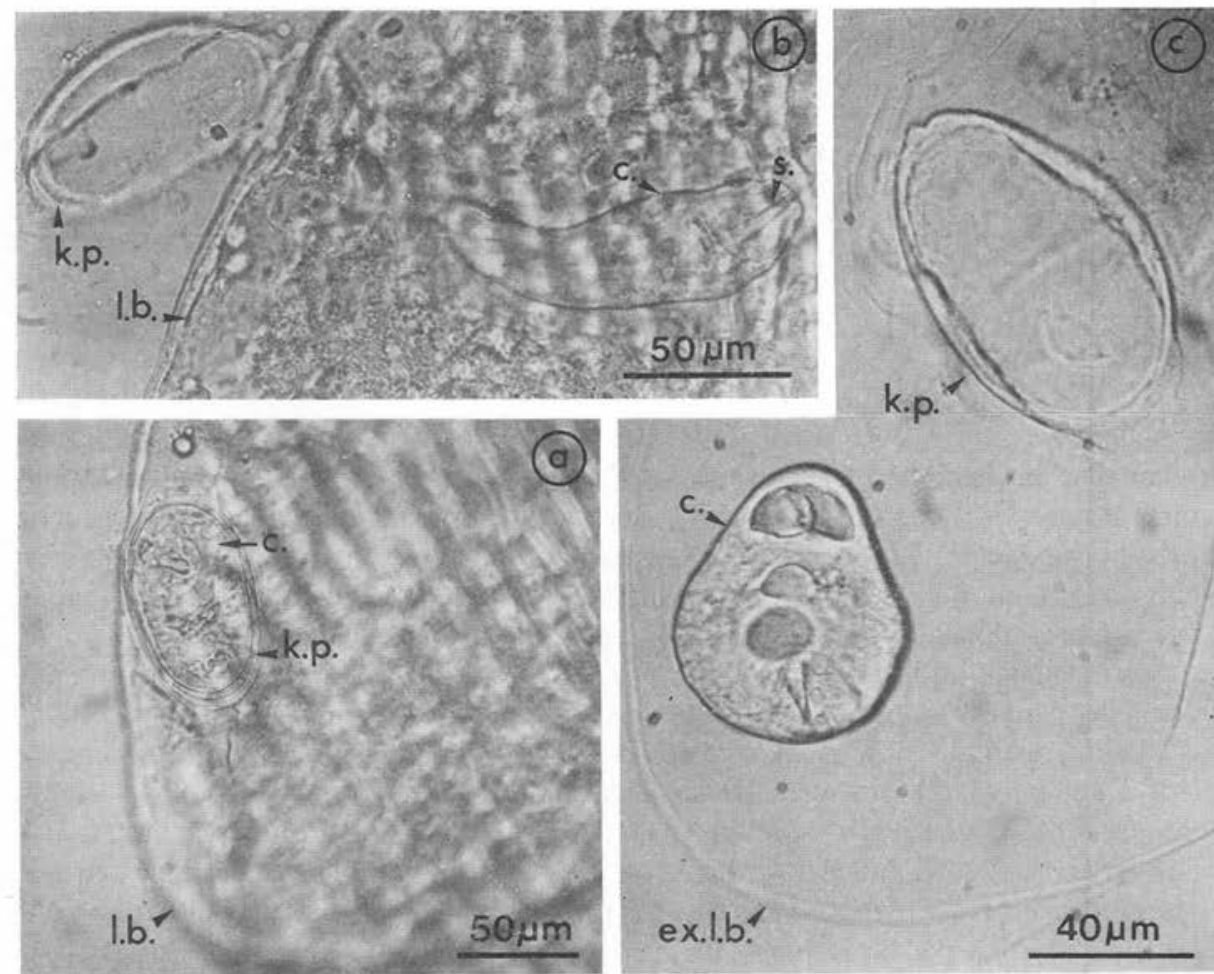

FIG. 2. - Pénétration de la cercaire

de Microphallus papillorobustus chez les Gammares.

a - Le kyste de pénétration (k.p.) accolé à la cuticule d'une lamelle branchiale (1.b.) abrite la cercaire décaudée (c.).

b - La cercaire (c.) s'élance dans la lamelle branchiale (1. b.). k.p. : kyste de pénétration; s. : stylet.

c - Impasse : hasard ou réaction de l'hôte, le Gammare a mué, la cercaire (c.). sortie de son kyste de pénétration (k.p.) est prisonnière de l'exuvie de la lamelle branchiale (ex. 1. b.).

Un pseudo-kyste est formé de manière similaire par la cercaire d'Acanthatrium oregonense (Lecithodendriidae) pénétrant dans les branchies de larves de Trichoptères. Face ventrale contre la cuticule branchiale, la cercaire produit presque instantanément une capsule en forme de dôme qui s'épaissit peu à peu (Burns, 1961).

La capsule ou pseudo-kyste, que l'on peut aussi appeler kyste de pénétration, remplirait quatre offices :

- la cercaire ainsi protégée évite d'être décrochée par le courant d'eau et les mouvements du Crustacé ;

- les produits émis par les glandes de pénétration ne sont pas dissous ;

- la larve peut prendre appui sur la paroi du kyste pour forer la cuticule et se propulser à l'intérieur de la lamelle branchiale ;

- la capsule adhérant à la cuticule prévient l'infestation secondaire par des germes pathogènes. 


\section{Rapports des métacercaires avec le tissu nerveux des gammares.}

Après pénétration et migration dans le corps, les larves de $M$. papillorobustus s'enkystent dans le tissu nerveux des Gammares, le plus fréquemment dans les ganglions cérébroïdes mais aussi, chez G. aequicauda, dans la chaîne nerveuse ventrale.

Dans la tête des Gammares, les ganglions cérébroïdes sont situés sur un plan parallèle au plan formé par l'insertion des deux paires d'antennes. De sorte qu'une section transversale de la tête offre une section frontale du cerveau et inversement.

Sur les coupes des ganglions deux types de territoires se distinguent nettement (Pl. I) :

- les neuropiles (masses denses de fibres nerveuses) ;

- les amas de cellules glomérulaires (cellules nerveuses pourvues de gros noyaux et situées à la périphérie des neuropiles (Madsen, 1960).

Le protocérébron comprend trois masses distinctes de neuropiles : les medulla terminalis, le corps central et les ganglions optiques (lamina ganglionaris, medulla externa et medulla interna). Le deutocerebron se compose des lobes accessoires, des lobes olfactifs en grappe, des lobes paraolfactifs et des neuropiles des antennes 1 . Le tritocerebron consiste en faisceaux de fibres des connectifs circum-œsophagiens, des nerfs tégumentaires et des antennes 2 (d'après Mac Pherson et Steele, 1980).

Chez Gammarus aequicauda et $G$. insensibilis une paire de statocystes est située dorsalement juste en arrière du rostre. Mac Pherson et Steele (1980), étudiant G. setosus signalent que les statocystes sont en relation avec le proto et le deutocérébron.

\section{Localisation des métacercaires.}

Des coupes sériées ont été effectuées sur les têtes de 21 Gammares parasités : 16 G. insensibilis, 5 G. aequicauda, mâles et femelles. Nous avons répertorié la position des métacercaires de $M$. papillorobustus chez tous les individus infestés par 1 ou 2 larves (4 G. aequicauda, 2 G. insensibilis) laissant de côté les sujets infestés par plus de 2 larves (15 individus).

Il existe couramment 10 kystes (au maximum 30) dans la tête d'un G. insensibilis ; ils occupent alors la quasi-totalité du volume de la tête et il est difficile d'appréhender leurs relations avec le tissu nerveux. En effet, eu égard à la dureté de la paroi kystique, les coupes de cerveau contenant de nombreuses métacercaires sont de mauvaise qualité; de plus, elles ne donnent aucune information sur la position préférentielle des larves.

Chez les 6 individus parasités par 1 ou 2 kystes, ceux-ci étaient situés dans le protocérébron, principalement dans les medulla terminalis (fig. 2). Une seule était logée au niveau du deutocérébron dans le neuropile d'une antennule.

Les métacercaires sont soit au sein des neuropiles, soit à la périphérie des ganglions cérébroïdes. Dans ce dernier cas, les coupes semi-fines prouvent que les kystes ne font pas protrusion dans le cerveau de l'extérieur mais que le neurilème (enveloppe du système nerveux) les recouvre ( $\mathrm{Pl} . \mathrm{Ib})$. 


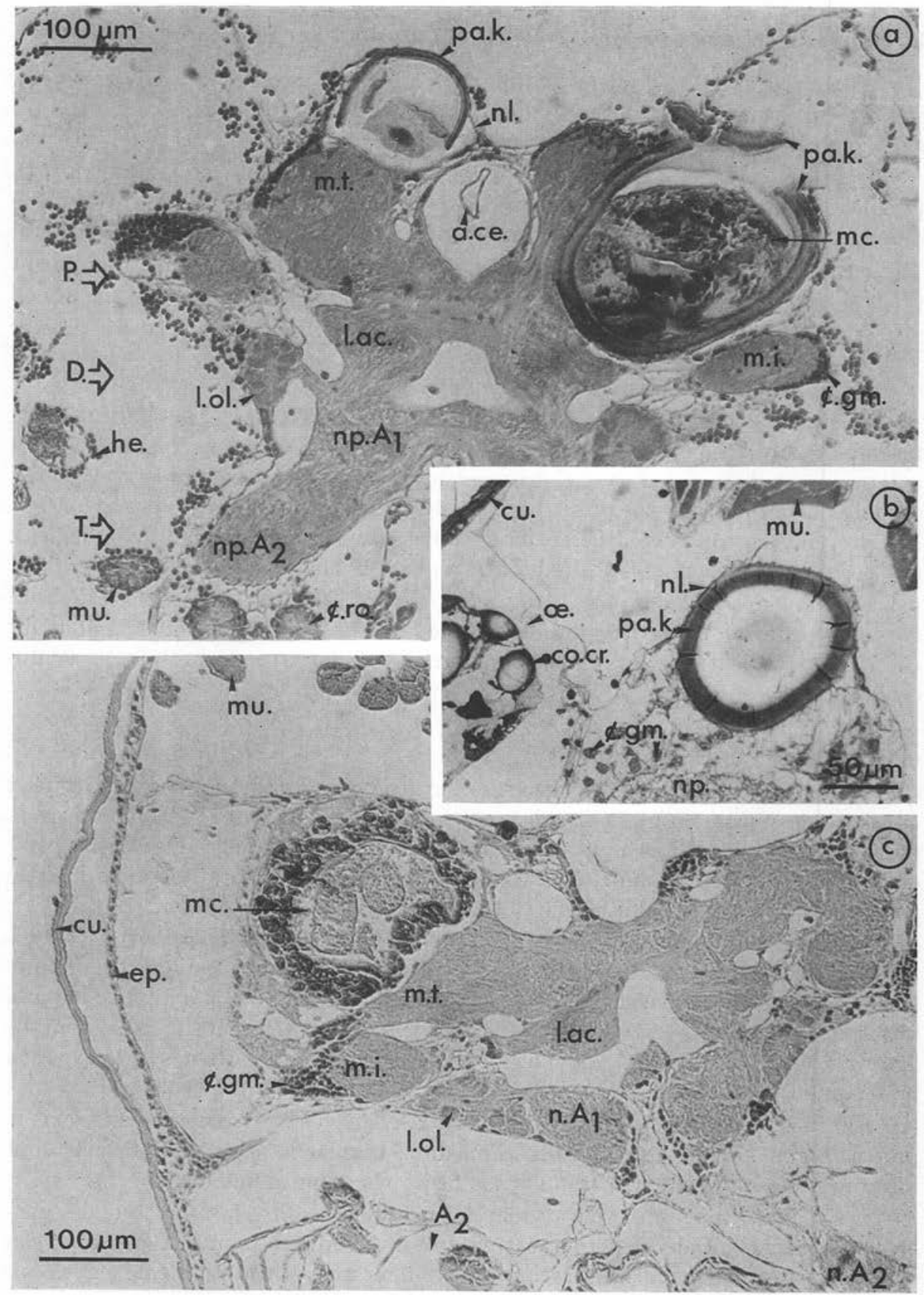

Planche I. 
Il est par ailleurs difficile de déterminer si le tissu nerveux est détruit et remplacé volume à volume ou s'il est seulement comprimé.

Chez G. insensibilis et $G$. aequicauda, les kystes cérébraux sont parfois encapsulés par un tissu brunâtre et granuleux. Les parasites sont atteints à différents stades de leur développement et à différents degrés. Tantôt la paroi kystique persiste et le parasite est vivant, tantôt la paroi kystique ayant disparu $(\mathrm{Pl}$. $\mathrm{Ic}$ ), le parasite est agonisant ou mort. Chez certains individus, on ne trouve plus dans les ganglions cérébroïdes que des amas pleins, granuleux et brunâtres, qui étaient sans doute initialement des métacercaires.

Des kystes de $M$. papillorobustus sont souvent localisés dans le mésosome, le métasome ou l'urosome de $G$. aequicauda, en la présence ou en l'absence de larves cérébrales. On les trouve ventralement, la plupart du temps à la naissance des coxopodites. Chaque fois que l'observation a été approfondie, ces kystes se sont révélés être situés sous le neurilème de la chaîne nerveuse ventrale ou d'un nerf métamérique (fig. 3).
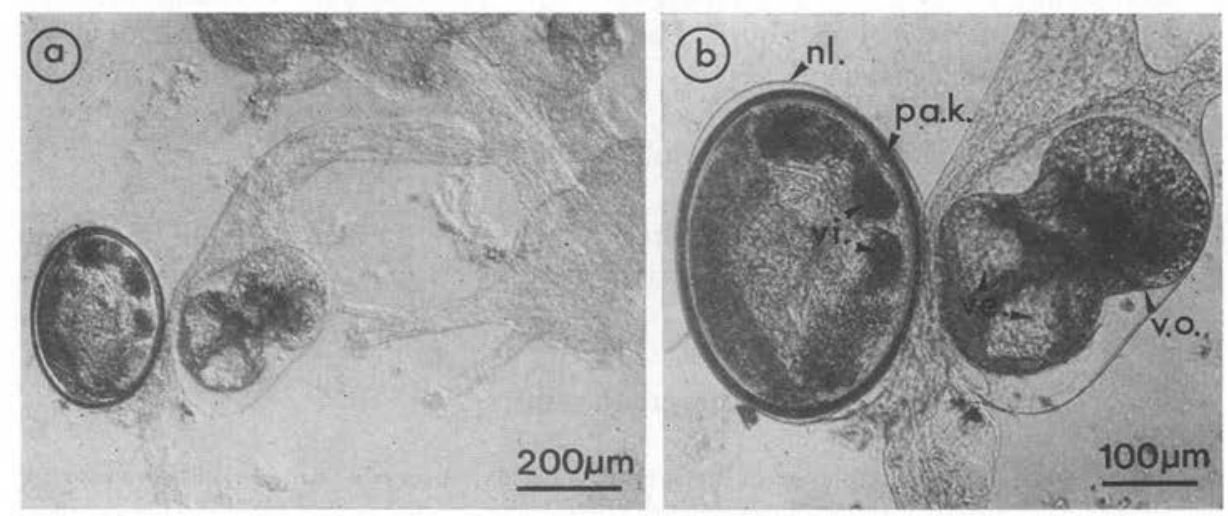

Fig. 3:- Métacercaires de Microphallus papillorobustus

en contact avec un nerf métamérique d'un Gammarus aequicauda.

a - Métacercaires, mature (à gauche) et en cours de maturation (à droite).

b - Détail de a. nl. : neurilème; v. o. : ventouse orale ; ve. : vessie ; vi. : vitellogènes ; pa.k. : paroi kystique.

Planche I. - Coupes de têtes de Gammares infestés par des métacercaires de Microphallus papillorobustus.

a - Coupe transversale d'une tête de G. aequicauda infesté par deux métacercaires situées dans le protocérébron.

b - Coupe semi-fine transversale d'une tête de G. aequicauda. La paroi kystique de la métacercaire située dans une medulla terminalis est enveloppée par le neurilème.

c - Coupe oblique (transverso-frontale) d'une tête de $G$. insensibilis. La métacercaire, située dans une medulla terminalis est encapsulée; sa paroi kystique a disparu.

P. : Protocérébron; D. : deutocérébron ; T. : Tritocérébron ; a. ce. : artère cérébrale ; c. gm. : cellules glomérulaires; c. ro. : cellules en rosette ; co. cr. : cône cristallin ; cu. : cuticule ; ep. : épiderme ; he. : hémocytes; 1 . ac. : lobe accessoire; 1. ol. : lobe olfactif; $n$. $\mathrm{A}_{1}$ : nerf antennulaire; $\mathrm{n} . \mathrm{A}_{2}$ : nerf antennaire; nl. : neurilème; np. : neuropile; np. $\mathrm{A}_{1}:$ neuropile antennulaire; np. $\mathrm{A}_{2}$ : neuropile antennaire ; $\mathrm{m}$. i. : medulla interna ; m. $\mathrm{t}$. : medulla terminalis ; mc. : métacercaire ; mu. : muscle ; œ. : œil; pa. k. : paroi kystique. 
L'étude d'échantillons de Gammarus insensibilis et de G. aequicauda infestés naturellement et expérimentalement a prouvé que la localisation de la larve de $M$. papillorobustus dans le cerveau ou bien dans la chaîne nerveuse ventrale de son hôte dépendait de l'espèce et de l'âge du Gammarus-hôte. Ces processus de sélection du site d'enkystement seront discutés dans un article ultérieur.

\section{Conclusion}

Les cercaires de $M$. papillorobustus infestent les Gammares en traversant la cuticule des lamelles branchiales; elles forment au préalable, à leur surface, des kystes de pénétration. Le stimulus-clef du déclenchement du processus de pénétration semble être un choc mécanique : les cercaires prises par le courant d'eau circulaire engendré par le battement des pléopodes sont projetées sur les lamelles branchiales ; aucun tactisme positif, chimiotactisme ou rhéotactisme, n'apparaît. Après migration les larves vont s'enkyster dans les ganglions cérébroïdes ou dans la chaîne nerveuse ventrale et les nerfs métamériques. Dans le microhabitat céphalique, il n'est pas rare de trouver une dizaine de kystes de $M$. papillorobustus. Cependant, quand il n'existe qu'une ou deux métacercaires, celles-ci sont situées au niveau du protocérébron principalement dans les medulla terminalis.

Remerciements : Nous tenons à remercier Monsieur le Professeur Louis Euzet qui a bien voulu nous faire part de ses conseils et de ses critiques.

\section{BIBLIOGRAPHIE}

BRUN B. : Variations intraspécifiques et spéciation chez deux espèces de Gammares d'eau saumâtre du groupe Gammarus locusta (Crustacés-Amphipodes). Thèse d'État, Provence, I65 p., I97I.

Burns W. C. : Penetration and development of Allassogonoporus vespertillionis and Acanthatrium oregonense (Trematoda : Lecithodendriidae) cercariae in caddis fly larvae. J. Parasit., 1961, $47,927-932$.

Macpherson B. R., Steele V. J. : Microanatomy of the central nervous system of Gammarus setosus Dementieva (Amphipoda). The supraoesophageal ganglion (Brain). Crustaceana, 1980, 38, II 3-I 20.

MADSEN N. : The brain of the amphipod Orchestia platensis Kroyer. Crustaceana, 1960, I, 173-1 78.

Prevot G. : Recherches sur le cycle biologique et l'écologie de quelques Trématodes nouveaux parasites de Larus argentatus michaellis Naumann dans le midi de la France. Thèse d'État, Aix-Marseille, 1974. 319 p.,

REBECQ J. : Recherches systématiques, biologiques et écologiques sur les formes larvaires de quelques Trématodes de Camargue. Thèse $d^{3} E$ Etat, Marseille, 1964, $222 \mathrm{p}$.

Srock J. H. : A revision of the European species of the Gammarus locusta group (Crustacea, Amphipoda). Zool. Verhand., 1967, 90, 1-56. 